\title{
Avaliação das Funções Cognitivas de Atenção, Memória e Percepção em Pacientes com Esclerose Múltipla
}

\author{
Evaluation of Cognitive Functions Related to Attention, Memory \\ and Perception of Patients with Multiple Sclerosis
}

\author{
José Osmar Frazão Freitas* \& Cilene Rejane Ramos Alves de Aguiar \\ Universidade Federal de Pernambuco, Recife, Brasil
}

\begin{abstract}
Resumo
Esclerose múltipla é uma doença desmielinizante, que leva à destruição de importantes estruturas do Sistema Nervoso Central. Essas lesões podem resultar em déficits cognitivos. Com o objetivo de investigar a relação entre esclerose múltipla e alterações cognitivas de atenção, memória e percepção, foram avaliados 28 pacientes em um Centro Estadual de Referência, que satisfaziam os critérios de inclusão: idade entre 20 e 55 anos, nível escolar mínimo de $2^{\circ}$ grau completo e classificação na Escala Expandida de Incapacidade menor que 7,0. Foram empregados quatro testes neuropsicológicos, validados pelo Conselho Federal de Psicologia: teste da cópia e reprodução de memória da figura complexa A de Rey-Osterrieth, teste de aprendizagem áudio-verbal de Rey, teste $\mathrm{d} 2$ e teste de dígitos. Para investigar o impacto da fadiga sobre as atividades cognitivas foi utilizada a escala MFIS-21Br. A análise estatística consistiu dos testes ANOVA, $t$ para amostras pareadas ou de Mann-Whitney e de Kolmogorov-Smirnoff, unicaudais à direita, para rejeição da hipótese nula com nível de significância de 0,05 . Os resultados apontaram perdas cognitivas com significância estatística nos pacientes com idade entre 40 e 55 anos e nos que apresentaram comprometimento por fadiga nos subdomínios cognitivo e psicossocial. Os pacientes com tempo de doença entre 5,0 e 19,9 anos apresentaram redução da pontuação nos testes, mas sem significância estatística. Concluiu-se que, dentre as funções cognitivas avaliadas, memória foi a mais freqüentemente comprometida, seguindo-se de atenção, sendo a percepção, comparativamente, a mais preservada.

Palavras-chave: Esclerose múltipla, déficits cognitivos, atenção, memória, percepção.
\end{abstract}

\begin{abstract}
Multiple sclerosis is a demyelinating disease that leads to the destruction of important structures of the Central Nervous System. These lesions may result in cognitive deficits. Aiming to investigate the relation between multiple sclerosis and cognitive alterations of attention, memory and perception 28 patients have been evaluated in a State Reference Center. They satisfied the following inclusion criteria: age between 20 and 55 years old, minimum scholarship level of High School and classification in the Expanded Disability Status Scale minor than 7.0. Four neuropsychological tests, validated by Federal Council of Psychology, were used: copy and memory reproduction of Rey-Osterrieth Complex Figure Test, Rey Audio-Verbal Learning Test, d2 Test and Digit-Span Test. To investigate the impact of fatigue on cognitive activities, MFIS-21Br scale was used. Statistical analysis consisted of ANOVA, pared samples $t$ test or Mann-Whitney test, and Kolmogorov-Smirnoff test, right sided, for rejection of null hypothesis with significance level of .05 . The results pointed out statistically significant cognitive losses for patients between 40 and 55 years old as well as for those who presented detriment due to fatigue in cognitive and psychosocial subdomains. Patients with disease duration between 5.0 and 19.9 years presented reduction on test scores, but without statistical significance. It was concluded that, among evaluated cognitive functions, memory was the most frequently compromised, followed by attention, and, perception, comparatively, was the most preserved. Keywords: Multiple sclerosis, cognitive deficits, attention, memory, perception.
\end{abstract}

Cognição pode ser compreendida como uma série de funções mentais que envolvem aquisição, armazenamento, retenção e uso do conhecimento. Esses processos incluem,

\footnotetext{
*Endereço para correspondência: Centro de Filosofia e Ciências Humanas, Departamento de Psicologia, Laboratório de Psicologia Experimental, Universidade Federal de Pernambuco, Rua Acadêmico Hélio Ramos, s/n, $9^{\circ}$ andar, Cid. Universitária, Recife, PE, Brasil 50670-901. E-mail: offreitas@hotmail.com
}

entre outros, os fundamentos da atenção, da percepção, da memória, do raciocínio e da aprendizagem. Através da execução dessas funções, o homem compreende e interage com o mundo, incluindo seus estímulos internos (pensamentos e sentimentos, entre outros), pois é por meio da capacidade cognitiva que se podem planejar ações, realizar julgamentos e solucionar problemas (Coltheart, 2004).

Com os recursos tecnológicos desenvolvidos nos últimos anos, é grande o interesse em identificar áreas 
cerebrais responsáveis por processos cognitivos, na expectativa de que a localização neural de funções psicológicas possa trazer mais informações (Capovilla, 2007). Para tanto, o neuropsicólogo inclui em sua metodologia de trabalho a avaliação dessas funções para direcionar a reabilitação de pacientes com perdas funcionais. Deve proceder à avaliação neuropsicológica, a qual tem por foco a análise das funções psicológicas associada a alterações neurais, no que difere da avaliação psicológica, cujo funcionamento é compreendido através do estudo comportamental (Mäder, 1996). Entre os objetivos da avaliação neuropsicológica estão: o auxílio ao diagnóstico diferencial, o estabelecimento da presença ou ausência de disfunções cognitivas, o acompanhamento de um quadro clínico e o encaminhamento do paciente para reabilitação (Boone, Lu, \& Wen, 2005). Esses conhecimentos sobre os distúrbios de memória, atenção e percepção motivaram estudos envolvendo pacientes com esclerose múltipla, porque eles apresentavam queixas de esquecimento e de dificuldades de concentração e lentificação do raciocínio, que prejudicavam a execução de tarefas laborais (Engel, Grein, \& Zettl, 2007). Essas pesquisas ganharam importância quando seus resultados foram correlacionados às características neuroanatômicas da doença.

A esclerose múltipla (EM) é uma doença inflamatória, crônica, desmielinizante e multicêntrica, que leva à destruição de estruturas importantes para o funcionamento do SNC (Ferreira et al., 2004), por processo auto-imune. Caracteriza-se por uma reação das células de defesa do sistema imunológico (linfócitos T), as quais, desconhecendo os lipídeos e as proteínas da bainha de mielina como próprias do indivíduo, a atacam e destroem, com ajuda dos macrófagos. A degeneração dos axônios impede a comunicação entre os neurônios pela impossibilidade da passagem do impulso elétrico, estabelecendo-se as incapacidades motoras e cognitivas do indivíduo (Goldacre, Wotton, Seagroatt, \& Yeates, 2004).

Pelo fato de a EM quase sempre comprometer, em seu início, a motricidade, assim como aspectos sensoriais da visão (nistagmo, alteração de campo visual, diplopia, escótomas, rebaixamento da acuidade visual, visão turva), estes sintomas e sinais têm sido priorizados nos estudos nacionais e internacionais (Gonzalez-Rosa et al., 2006). No entanto, nas últimas décadas, se tem investigado o comprometimento cognitivo associado à EM, principalmente após a determinação de uma prevalência estimada de $40 \%$ a $65 \%$ (Amato et al., 2006). Com muita freqüência, esse comprometimento acomete aprendizagem, memória, velocidade de processamento da informação, discriminação visual espacial, fluência verbal, raciocínio abstrato e funções de execução, causando um prejuízo a $70 \%$ a $80 \%$ dos pacientes com esclerose múltipla (Roessler \& Rumrill, 2003). O comprometimento neuropsicológico, envolvendo percepção, atenção, reconhecimento, inteligência, imaginação, memória, planejamento e julgamento, é considerado "um sintoma sutil" frente às manifestações motoras e sensoriais da esclerose múltipla e, segundo Winkelmann, Engel, Apel e Zettl (2007), foi negligenciado durante muito tempo.

A disfunção cognitiva é tipicamente detectada tardiamente no curso da esclerose múltipla, embora possa compor sintomas iniciais em $26 \%$ a $54 \%$ dos pacientes, mostrando estagnação ao longo do tempo (Rogers \& Panegyres, 2007). Amato et al. (2006), acompanhando a história natural da disfunção cognitiva em 45 pacientes, com segmento de dez anos, identificaram que, no estágio inicial da doença, a disfunção cognitiva estava ausente em $74 \%$ dos pacientes, era discreta em $8 \%$ e moderada em $18 \%$, percentuais que se alteraram para $44 \%, 34 \%$ e $22 \%$, após dez anos de doença. Dentre as funções cognitivas, a mnemônica é o domínio mais freqüentemente acometido, identificado em $40 \%$ a $60 \%$ dos portadores de esclerose múltipla, com variação em função do teste empregado para sua aferição (Moriarty et al., 1999; Negreiros, Mattos, Paes, Landeira-Fernandez, \& Alvarenga, 2008). Quanto à atenção nos quadros de EM, tem-se demonstrado repetidamente uma capacidade reduzida de manter e manipular informações na mente, processos comumente conceituados como funções de memória de trabalho ou de atenção concentrada. Estudos têm demonstrado que os prejuízos de processamento podem ocorrer no início da doença e contribuir para a disfunção de memória e de raciocínio abstrato (Schulz, Kopp, Kunkel, \& Faiss, 2006).

Diante dessas evidências, Benedict e Zivadinov (2007) sugerem a necessidade de avaliar o comprometimento neuropsicológico e, em especial, o cognitivo de portadores de EM antes da consulta médica e por profissionais não médicos, para permitir a instituição de medidas que possam auxiliar o portador dessa enfermidade a ter melhor qualidade de vida. Benedict e Zivadinov (2007) julgam também que, independente do teste a ser empregado, essa avaliação é essencial porque outros fatores como depressão, ansiedade, estresse e fadiga, podem contribuir ou mesmo mimetizar prejuízos cognitivos.

As funções cognitivas cuja perda representa maior impacto na qualidade de vida dos pacientes são as de percepção, atenção e memória (Penner, Kappos, Raush, Opwis, \& Radü, 2006). A preservação das três funções a serem avaliadas nos pacientes de EM assume um caráter de maior relevância porque, além de possibilitar sua adequação às atividades profissionais e sociais, permite maior compreensão e chance de execução das rotinas e orientações exigidas no tratamento.

O diagnóstico e o tratamento da EM exigem um Centro Estadual de Referência com assistência interprofissional a portadores de doenças desmielinizantes, dada a complexidade e a necessidade do uso de medicações especiais de alto custo. Dois aspectos da EM devem ser enfatizados para que se compreendam os objetivos de um Centro de Referência: o primeiro se refere ao acometimento de indivíduos predominantemente na faixa etária dos 20 aos 55 anos, portanto economicamente pro- 
dutivos, o que confere à doença a característica de se constituir em agravo socioeconômico; em segundo lugar, o alto custo do tratamento, pois além da medicação, a terapêutica inclui sua locomoção para atendimento, exames por imagem e laboratoriais e adaptações nos ambientes físicos freqüentados pelos pacientes (Ferreira et al., 2004).

No Hospital da Restauração (HR), na cidade do Recife, funciona o Centro Estadual de Referência para Atenção a Pacientes Portadores de Doenças Desmielinizantes (CRAPPDD), que tem, dentre seus objetivos, a assistência multiprofissional a 552 portadores de doenças desmielinizantes, com seguimento de até 20 anos (Ferreira et al., 2004). Apesar de essa assistência vir sendo prestada desde 1987, a autorização para que os pacientes neurológicos tivessem acesso à medicação de alto custo ocorreu no início de 1996, levando à modernização do atendimento. Apesar desses avanços, a avaliação das funções cognitivas não tem sido contemplada, o que motivou a escolha do tema para a atual investigação.

A presente pesquisa buscou investigar a relação entre a EM e as alterações cognitivas de percepção, atenção e memória dos pacientes atendidos no Centro Estadual de Referência para Atenção a Pacientes Portadores de Doenças Desmielinizantes, utilizando-se, para tanto, testes neuropsicológicos.

\section{Método}

\section{Participantes}

Participaram deste estudo 28 pacientes atendidos em regime ambulatorial, por busca espontânea ou por referência de outros serviços de saúde, portadores de esclerose múltipla, no período de Junho a Outubro de 2008. Foram admitidos como critérios de inclusão: idade entre 20 e 55 anos no momento da coleta; nível escolar mínimo de $2^{\circ}$ grau completo, para que a baixa escolaridade não atuasse como fator de confundimento no desempenho dos testes psicológicos e EDSS menor que 7,0, para que a incapacidade física não impossibilitasse o paciente de realizar qualquer dos testes.

Considerando que, no local do estudo, havia 327 portadores de esclerose múltipla, cadastrados e elegíveis para o estudo, porque obedeciam aos critérios de inclusão, para o cálculo do tamanho amostral admitiu-se: precisão desejada de $18 \%$, prevalência de distúrbios cognitivos em $54 \%$ e efeito de desenho igual a 1, em nível de significância de $5 \%$. Resultou um tamanho amostral de 28 pacientes, os quais foram selecionados por tábua de números aleatórios a partir de lista nominal.

Os 28 pacientes investigados caracterizaram-se por: predomínio do gênero feminino, numa proporção 2,5:1, em relação ao masculino; média de idade igual a $37,9 \pm$ 1,8 anos, variando de 20 a 55 anos, estando $75 \%$ com 30 anos ou mais; escolaridade igualmente distribuída entre ensino médio e superior, completo ou incompleto, mais freqüentemente com estado civil solteiro ou casado e de- sempenhando ocupações nos setores técnico de comércio ou de nível superior. Quanto à esclerose múltipla, o tempo de doença médio igualou-se a $8,6 \pm 0,7$ anos, variando entre 2,5 e 16,4 anos com predomínio de cinco ou mais anos, forma clínica surto/remissão (SR) e gravidade leve pela Escala Expandida de Incapacidade. Foram menos frequentes casos na forma secundariamente progressiva (SP).

\section{Instrumentos}

Foram empregados cinco instrumentos para coleta de dados. Com a escala de fadiga Modified Fatigue Impact Scale (MFIS-21Br), validada para o idioma Português Brasil por Pavan et al. (2007), avaliaram-se os efeitos da fadiga sobre as atividades físicas, cognitivas e psicossociais, categorizados em ausente, com pontuação total menor que 38 pontos; baixo, quando entre 39 a 58 , e alto impacto, quando maior que 59 pontos (The Consortium of Multiple Sclerosis Centers, 1997).

O Teste da Cópia e de Reprodução de Memória da Figura Complexa A de Rey-Osterrieth (ROCF), permitiu avaliar percepção visual, organização visuoespacial pelos resultados da cópia; reprodução de memória de trabalho (decorridos no máximo três minutos do término da cópia, denominada $1^{\mathrm{a}}$ memória) e reprodução de memória de curto prazo (decorridos 30 minutos da cópia, denominada $2^{\mathrm{a}}$ memória). Percentil de pontos menor que 50 foi adotado como ponto de corte para caracterizar perda em cada uma das fases do teste (Rey, 1999).

O Teste de Aprendizagem Áudio-Verbal de Rey (RAVLT) foi empregado para avaliação de memória de trabalho, após a primeira leitura, aprendizagem (analisada pela soma de acertos das cinco leituras), interferência pró-ativa (analisada pela razão entre acertos após interferência e após quinta leitura) e memória verbal de curto prazo, (avaliada pelos acertos após 20 minutos) e interferência retroativa (avaliada pela razão entre a última tentativa e aquela após interferência). A interferência pró-ativa foi considerada como sendo aquela que ocorre quando uma nova informação impede a retenção de uma aprendizagem anterior, enquanto que se admitiu por interferência retroativa a redução de retenção de informações previamente aprendidas causada por aprendizagens subseqüentes (Griffiths et al., 2005).

Com o Teste $\mathrm{d} 2$ foram avaliadas atenção concentrada (pelo percentil de resultado líquido) e atenção sustentada (avaliada pela distribuição das médias de resultado bruto, total de erros e percentual de erros nas três partes do teste), independente da inteligência, obedecendo aos critérios de Brickenkamp (2000).

O Teste de dígitos em ordem direta e inversa (Digit Span) para avaliação de memória de trabalho auditiva (avaliada pelo total de pontos da ordem direta), memória operacional (avaliada pelo total de pontos da ordem inversa) e atenção (pela diferença entre os pontos da ordem direta e inversa), obedeceu ao método de Kaplan, Fein, Morris e Delis (1991). 
Os resultados dos testes foram entregues ao neurologista responsável, para compor o prontuário do paciente. Foram consideradas as variáveis independentes, que podiam interferir sobre os resultados dos testes empregados, relativas a: idade, gênero, escolaridade, tempo de doença expressa em anos, forma clínica segundo os critérios de Poser et al. (1983) e modificado de McDonald et al. (2001), incapacidade avaliada pela Escala Expandida de Incapacidade Funcional (EDSS), idealizada por Kurtzke e Page (1997), categorizada em leve, com EDSS entre 0,0 e 3,5, moderada, com EDSS entre 4,0 e 6,5 e severa, com EDSS igual a 7,0.

\section{Realização da Pesquisa}

Iniciou-se uma entrevista para caracterização das informações sócio-demográficas, em sala especialmente destinada para esta finalidade. Os testes foram aplicados pelo pesquisador, na seguinte ordem: MFIS-21Br, figura complexa A de Rey-Osterrieth, teste de aprendizagem áudio-verbal de Rey, teste $\mathrm{d} 2$ e teste de dígitos em ordem direta e inversa, respeitando-se os tempos de aplicação de cada um deles conforme procedimentos de coleta, anotando as observações feitas pelo paciente durante as respostas.

\section{Análise Estatística}

Utilizando o programa EPI-INFO, versão 6.04d, do Center for Disease and Prevention, da Organização Mundial da Saúde, foi construído o banco de dados, posteriormente convertido para o formato compatível com o programa Statistical Package for Social Sciences (SPSS), versão 13.0, com o qual foi feito o tratamento estatístico dos dados. Foram calculados os parâmetros estatísticos de média e erro padrão da média das variáveis: idade, tempo de doença, percentuais de fadiga total, cognitiva e psicossocial do teste MFIS-21Br, percentuais da figura complexa de Rey-Osterreith, pontuações dos testes d2 e de dígitos. Foram empregados teste ANOVA (para resultados do teste MFIS-21Br e da aprendizagem auditiva verbal de Rey), teste $t$ para amostras pareadas (resultados do teste de dígitos e das variações do teste $\mathrm{d} 2$ ), teste de Mann-Whitney (percentis dos testes de aprendizagem auditiva verbal de Rey, teste de dígitos e teste $\mathrm{d} 2$, segundo variáveis da EM) e Teste de Kolmogorov-Smirnoff (percentis da soma de pontos nas três etapas da Figura Complexa de Rey-Osterrieth, segundo variáveis da esclerose múltipla), para comparação entre grupos de idade, forma clínica, gravidade avaliada pelo EDSS e tempo de doença, todos em nível de significância igual a 0,05, unicaudais à esquerda, para rejeição da hipótese nula, posto que apenas a redução das pontuações tem valor clínico.

O projeto de pesquisa foi aprovado pelo Comitê de Ética em Pesquisa com Seres Humanos do Hospital da Restauração, com o registro $n^{\circ}$. CAAE $n^{\circ}$. 0007.0.102.102-08.

\section{Resultados}

\section{Avaliação da Fadiga pelo Questionário MFIS-21Br}

Pelo questionário MFIS-21Br, constatou-se que a média dos percentuais de fadiga igualou-se a: 41,6 $\pm 3,4$ pontos percentuais para fadiga total, variando entre $7 \% \mathrm{e}$ $78 \% ; 20,4 \pm 1,6$ pontos percentuais no subdomínio físico, $17,5 \pm 1,8$ pontos percentuais no subdomínio cognitivo e $3,8 \pm 0,5$ pontos percentuais no psicossocial. O maior comprometimento por fadiga ocorreu no subdomínio físico.

Considerando os pontos de corte para classificação de fadiga recomendados pelo The Consortium of Multiple Sclerosis Centers (1997), identificou-se que o grau alto de fadiga mais freqüentemente ocorreu em pacientes com comprometimento nos três subdomínios do MFIS-21Br, ou quando a fadiga física e cognitiva foram percebidas. A presença de fadiga cognitiva predominou nos pacientes com comprometimento leve ou ausente. No entanto a fadiga psicossocial, apesar de ser menos freqüente que a física ou a cognitiva, esteve presente em pacientes com grau alto de comprometimento ([5/6 casos $=83,3 \%]$, Tabela 1$)$.

Tabela 1

Distribuição dos 28 Portadores de Esclerose Múltipla Segundo Presença de Fadiga nos Subdomínios do MFIS-21Br

\begin{tabular}{lccc}
\hline Subdomínio(s) afetado(s) pela fadiga & & Grau de fadiga & \\
\cline { 2 - 4 } & Ausente & Baixo & Alto \\
\hline Físico, cognitivo e psicossocial & - & $1(3,6 \%)$ & $5(17,9 \%)$ \\
Físico e psicossocial & $1(3,6 \%)$ & $1(3,6 \%)$ & - \\
Físico e cognitivo & - & $1(3,6 \%)$ & $1(3,6 \%)$ \\
Cognitivo e psicossocial & - & $2(7,1 \%)$ & - \\
Físico & $3(10,7 \%)$ & $2(7,1 \%)$ & - \\
Cognitivo & $2(7,1 \%)$ & - & - \\
Psicossocial & $1(3,6 \%)$ & - & - \\
Ausente nos três domínios & $6(21,4 \%)$ & $9(32,1 \%)$ & $6(21,4 \%)$ \\
\hline Totais & $13(46,4 \%)$ & & \\
\hline
\end{tabular}


Considerando médias e erros padrão da média das pontuações percentuais do domínio e dos subdomínios do MFIS-21Br, segundo as variáveis da esclerose múltipla, observou-se que os pacientes na forma clínica secundária progressiva tiveram maior comprometimento por fadiga física $(28,5 \pm 5,5$ contra $19,7 \pm 1,6$ para a forma SR) e no domínio total $(47,0 \pm 16,0$ contra $41,2 \pm 3,5$ na forma SR), ambas sem significância. A gravidade da EM, avaliada pela EDSS, não interferiu na fadiga; os pacientes na faixa etária de 40 a 55 anos tiveram maior comprometimento por fadiga em todos os subdomínios, sendo significante apenas no psicossocial $(p=0,045)$; o maior tempo de doença acarretou maior fadiga, em todos os subdomínios, com possível significância no domínio total $(p=0,070)$ e no físico $(p=0,056)$.

\section{Resultados do Teste da Figura Complexa A de Rey-Osterrieth}

Avaliando os percentis do total de pontos obtidos no teste da figura complexa A de Rey-Osterrieth pelos 28 pacientes, observou-se que $60,7 \%$ obtiveram $75 \%$ a $100 \%$ do total de pontos da cópia, mas, no desenho feito de memória logo após a cópia ( $1^{\mathrm{a}}$ memória) e decorridos 30 minutos da cópia ( $2^{\mathrm{a}}$ memória), a maioria dos pacientes atingiu entre zero e $25 \%$ do total geral de pontos (Figura 1).

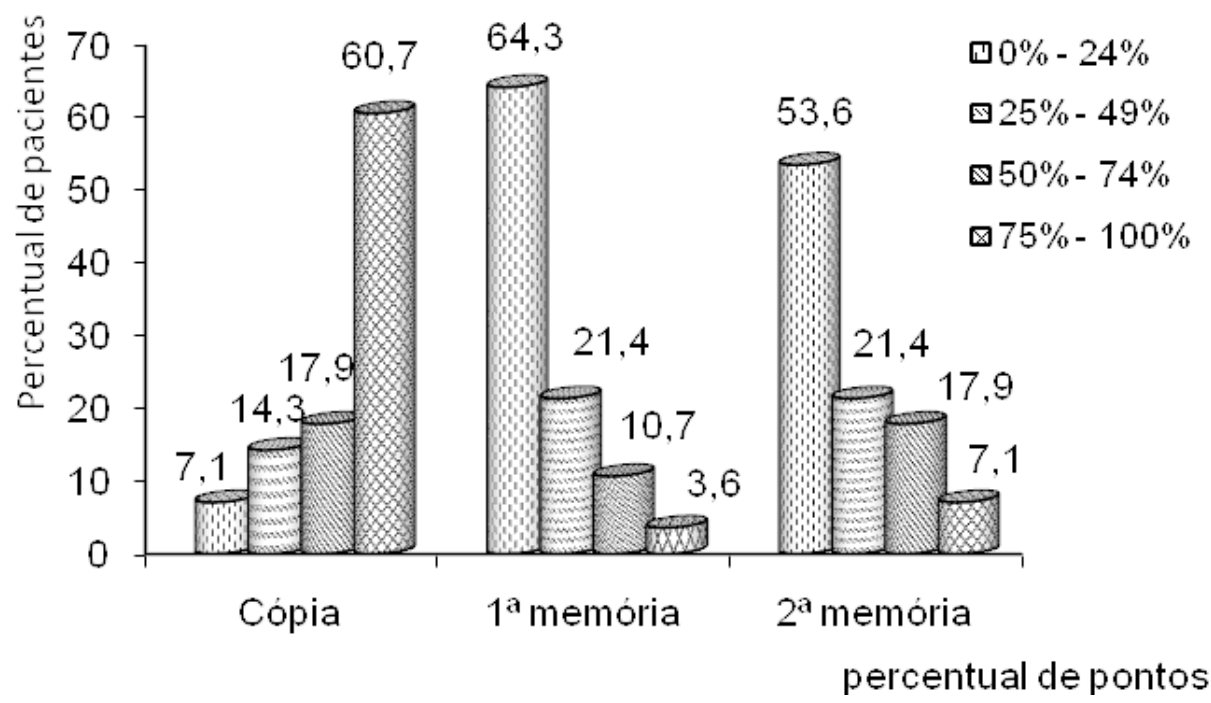

Figura 1. Distribuição das três fases da Figura Complexa de Rey-Osterrieth segundo percentual de pontos obtidos por 28 portadores de esclerose múltipla.

Observou-se que 89,3\% dos pacientes apresentaram redução dos pontos entre a cópia da figura complexa de Rey-Osterrieth e o desenho da primeira memória e 17,9\% tiveram diminuição dos pontos entre a $1^{\mathrm{a}}$ memória e a $2^{\mathrm{a}}$ memória. Comparando esses três momentos do teste, identificou-se que a maior redução de pontos ocorreu entre a cópia e $1^{\mathrm{a}}$ memória (memória de trabalho).

Para detalhar essas perdas, foram analisadas as variações de percentis de pontos obtidos na Figura Complexa de Rey-Osterrieth por 28 pacientes, segundo as variáveis da esclerose múltipla, identificando-se perdas significantes de memória de trabalho $(p=0,032)$ e de curto prazo $(p=0,027)$ dos pacientes na faixa etária de 40 a 55 anos. Constatou-se que pacientes com: EM na forma surto/remissão, de gravidade leve avaliada pelo EDSS, presença de fadiga cognitiva ou psicossocial e tempo de doença maior que 4,9 anos tiveram pontuações menores de memória de trabalho e de curto prazo, quando comparados àqueles na forma secundariamente progressiva, com gravidade moderada, sem fadiga cognitiva ou psicossocial e com menos de cinco anos de doença, res- pectivamente. No entanto essas diferenças não foram significantes.

Resultados do Teste de Aprendizagem Auditiva Verbal de Rey

Os resultados dos parâmetros de avaliação do teste de aprendizagem auditiva verbal de Rey (RAVLT) dos 28 portadores de esclerose múltipla permitiram identificar aumento significante de acertos entre a quinta leitura e a primeira leitura $(p<0,001)$, entre a quinta leitura e os acertos após interferência $(p=0,026)$ e o percentual de acertos do reconhecimento decorridos 20 minutos e a quinta leitura $(p<0,001)$.

Classificando os portadores de esclerose múltipla segundo os critérios de Andersson et al. (2006), constatouse que $53,6 \%$ dos pacientes não apresentaram perda de memória, avaliada pelo RAVLT, enquanto que para 17,9\% houve perda severa e $28,6 \%$, perda moderada.

Distribuindo os pacientes de acordo com o diagnóstico de perda de memória avaliada pelo teste de aprendizagem auditiva verbal de Rey, constatou-se que apenas as variáveis de presença de fadiga psicossocial $(p=0,004)$ e 
fadiga global $(p=0,026)$ interferiram sobre a perda no teste, com significância estatística.

\section{Resultados do Teste d2}

Nos parâmetros de avaliação do teste $\mathrm{d} 2$, houve pontuações baixas de resultados brutos e líquidos, resultados altos de total de erros e baixos percentis para os parâmetros de avaliação relativos a resultado bruto, resultado líquido e total percentual de erros.

Quanto à atenção concentrada avaliada por meio do percentil do resultado líquido do teste $\mathrm{d} 2$, constatou-se que pacientes com a forma clínica secundariamente progressiva, grau moderado de incapacidade avaliada pelo EDSS, com mais de 39 anos de idade, apresentando fadiga física e com tempo de doença igual ou maior que cinco anos tiveram percentis menores. No entanto a única diferença com significância foi a relativa à idade $(p=0,038)$.

Avaliando a atenção sustentada por meio das variações no decorrer do teste d2, observou-se sua manutenção configurada pelo aumento da média de resultados brutos, sem diferença significante entre a primeira parte e a segunda $(p=0,055)$, e entre a segunda e a terceira partes $(p=0,130)$. Identificou-se também redução não significante do total médio de erros entre a primeira e a segunda parte do teste $(p=0,314)$, com subseqüente aumento, também sem significância $(p=0,548)$. O mesmo comportamento constatou-se no percentual médio de erros, com diferenças não significantes entre a primeira parte e a segunda $(p=0,140)$ e entre a segunda e a terceira $([p=0,727]$, Tabela 2$)$.

Tabela 2

Distribuição de 28 Portadores de Esclerose Múltipla, Segundo Variáveis do Teste d2

\begin{tabular}{lccc}
\hline Variáveis & \multicolumn{3}{c}{ Variação nas partes do teste d2 } \\
\cline { 2 - 4 } & $1^{\mathrm{a}} \mathrm{a} 4^{\mathrm{a}}$ linhas & $5^{\mathrm{a}}$ a $10^{\mathrm{a}}$ linhas & $11^{\mathrm{a}}$ a $14^{\mathrm{a}}$ linhas \\
\hline Resultado bruto médio & $26,01 \pm 1,54$ & $27,71 \pm 1,48$ & $28,52 \pm 1,56$ \\
Total médio de erros & $6,07 \pm 0,37$ & $5,93 \pm 0,37$ & $6,00 \pm 0,41$ \\
Percentual médio de erros & $30,29 \pm 5,08$ & $25,13 \pm 2,78$ & $25,53 \pm 3,35$ \\
\hline
\end{tabular}

Nota. Os valores de $p$ referem-se ao teste $t$ para amostras pareadas.

Resultados do Teste de Dígitos

No teste de dígitos foi possível constatar a existência de redução significante de pontos da ordem inversa, quando comparados à direta, e essa diferença foi significante $(p<0,001)$. Constatou-se que $25(89,3 \%)$ pacientes obtiveram pontuação em ordem inversa menor que na direta, um $(3,6 \%)$ paciente conseguiu pontuação igual em ambas as ordens e dois $(7,1 \%)$ tiveram pontuação na ordem inversa maior que na ordem direta.

Adotado o critério de Figueiredo e Nascimento (2007) de discrepância entre os dígitos da ordem direta e os da ordem inversa entre dois e três como normalidade de memória operacional, identificou-se que seis $(21,4 \%)$ pacientes apresentaram uma diferença de pontuação menor que dois pontos, enquanto que para 12 (42,9\%), a diferença esteve entre dois e três pontos e, em 10 $(35,7 \%)$, foi maior que três pontos, na ordem inversa quando comparada à direta. As perdas identificadas ao teste de dígitos não se associaram a qualquer das variáveis da esclerose múltipla.

\section{Sintese dos Resultados dos Testes Neuropsicológicos Segundo Variáveis da Esclerose Múltipla}

Na Tabela 3, está apresentada a síntese das associações entre as variáveis da esclerose múltipla e os resultados dos testes neuropsicológicos, para os quais se observou redução de pontuação, independente de significância estatística.

\section{Discussão}

Este estudo permitiu comprovar que pelo menos uma das funções de atenção, memória e percepção esteve comprometida em todos os pacientes, independente da forma clínica (Tabela 3). Esses resultados parecem respaldar a recomendação de que pacientes com EM devem ser submetidos à avaliação cognitiva, porque as perdas podem estar presentes, mesmo na forma clínica surto/remissão, desde o início da doença (Benedict \& Zivadinov, 2006).

Mais da metade dos pacientes apresentou perda de atenção avaliada pelo teste $\mathrm{d} 2$ (Tabela 3 ). Verificou-se redução significante da atenção concentrada em pacientes com mais de 39 anos de idade, constatação referida por Schulz et al. (2006) quando submeteram 31 pacientes à bateria computadorizada attention test battery (TAP) e identificaram que 33\% deles tinham perda de atenção. No entanto empregaram testes diferentes e não associaram os resultados aos parâmetros da esclerose múltipla, como no presente estudo.

Quanto à atenção sustentada, avaliada pela comparação de desempenho nas três partes do teste $\mathrm{d} 2$, não se verificou perda significante (Tabela 2). Balsimelli, Mendes, Bertolucci e Tilbery (2007) analisaram o prejuízo de atenção de 115 pacientes com EM na forma SR, por meio de uma bateria composta pelos testes de dígitos, trilhas, teste de cancelamento e stroop. Concluíram haver redu- 
Freitas, J. O. F. \& Aguiar, C. R. R. A. (2012). Avaliação das Funções Cognitivas de Atenção, Memória e Percepção em Pacientes com Esclerose Múltipla.

Tabela 3

Síntese das Associações entre Variáveis da Esclerose Múltipla e Resultados dos Testes Neuropsicológicos

\begin{tabular}{|c|c|c|c|c|c|c|}
\hline \multirow[t]{2}{*}{ Variáveis da EM } & $\begin{array}{l}\text { ROCF } \\
\text { cópia }\end{array}$ & $\begin{array}{c}\text { ROCF } \\
1^{\mathrm{a}} \text { memória }\end{array}$ & $\begin{array}{c}\text { ROCF } \\
2^{\mathrm{a}} \text { memória }\end{array}$ & $\begin{array}{l}\text { RALVT } \\
\text { A7 e A1 }\end{array}$ & D2 & $\begin{array}{c}\text { Dígitos } \\
\text { diferença } \\
\text { direta-inversa }\end{array}$ \\
\hline & $\begin{array}{c}\text { Percepção visual, } \\
\text { organização } \\
\text { visuoespacial }\end{array}$ & $\begin{array}{l}\text { Memória } \\
\text { de trabalho }\end{array}$ & $\begin{array}{c}\text { Memória } \\
\text { de curto prazo }\end{array}$ & $\begin{array}{c}\text { Memória } \\
\text { de curto prazo }\end{array}$ & $\begin{array}{c}\text { Atenção } \\
\text { concentrada/ } \\
\text { sustentada }\end{array}$ & $\begin{array}{c}\text { Memória } \\
\text { operacional }\end{array}$ \\
\hline $\begin{array}{l}\text { Pacientes com perda }(\%) \\
\text { Forma Clínica }\end{array}$ & 21,4 & 89,2 & 85,7 & 17,8 & 57,1 & 57,1 \\
\hline $\begin{array}{l}\text { Surto/remissão } \\
\text { Secundária progressiva }\end{array}$ & $X$ & $\mathrm{X}$ & $X$ & & $\mathrm{X}$ & $\mathrm{X}$ \\
\hline $\begin{array}{l}\text { Gravidade (EDSS) } \\
\text { moderada }\end{array}$ & $\mathrm{X}$ & & & & $\mathrm{X}$ & $\mathrm{X}$ \\
\hline $\begin{array}{l}\text { Idade } \\
\quad 20-39\end{array}$ & & & & & & \\
\hline $40-55$ & & $\downarrow$ & $\downarrow$ & $\downarrow$ & $\downarrow$ & \\
\hline Fadiga & & & & & & \\
\hline $\begin{array}{l}\text { fisica presente } \\
\text { cognitiva presente }\end{array}$ & $\mathrm{X}$ & $\mathrm{X}$ & $\mathrm{X}$ & $\begin{array}{l}X \\
X\end{array}$ & $\mathrm{X}$ & \\
\hline $\begin{array}{l}\text { psicossocial presente } \\
\text { total presente }\end{array}$ & & $\mathrm{X}$ & $\mathrm{X}$ & $\downarrow$ & & $\mathrm{X}$ \\
\hline $\begin{array}{l}\text { Tempo de doença } \\
5,0-19,9\end{array}$ & & & & & & \\
\hline & $\mathrm{X}$ & $\mathrm{X}$ & $\mathrm{X}$ & $\mathrm{X}$ & $\mathrm{X}$ & \\
\hline
\end{tabular}

Nota. Legenda: X - redução da pontuação no teste, sem diferença estatisticamente significante; $\downarrow$ - redução da pontuação no teste, com significância estatística.

ção de atenção, ao identificarem que os sujeitos analisados necessitavam de um tempo maior para completar cada um dos testes, especialmente o stroop, que avalia atenção sustentada. No entanto a similaridade de resultados entre o presente estudo e outras pesquisas deve ser considerada com cautela porque os testes empregados e os delineamentos dos estudos são diferentes. Engel et al. (2007), na Alemanha, e Arnett e Forn (2007), na Espanha, chamaram a atenção para esse problema metodológico e propuseram baterias de testes neuropsicológicos para avaliação de disfunção cognitiva em pacientes com EM. Apesar de reconhecerem a necessidade de padronizar essa avaliação, recomendaram baterias diferentes.

Também no Brasil se tem buscado o conjunto mais adequado de testes neuropsicológicos para avaliação dos prejuízos da esclerose múltipla. Noronha, Sisto, Bartholomeu, Lamounier e Rueda (2006) propuseram o emprego dos testes AC (atenção concentrada) e AS (atenção sustentada); Negreiros et al. (2008) sugeriram uma bateria composta por nove testes. Essas tentativas vêm comprovar que ainda não há consenso quanto aos testes mais adequados para tal avaliação. O presente trabalho pode se constituir em mais uma tentativa nessa busca, pois tem como diferencial o emprego do teste $\mathrm{d} 2$, investigando atenção concentrada e sustentada.

Quanto à avaliação dos prejuízos de memória, constatou-se que $89,2 \%$ dos pacientes tinham perda na memória de trabalho e $85,7 \%$, na memória de curto prazo, ambas avaliadas pela Figura Complexa de Rey-Osterrieth (Figura 1); 57,1\% tinham prejuízo da memória operacional, avaliada pela diferença do teste de dígitos em ordem direta e inversa, e 17,8\% tinham perda na memória de curto prazo avaliada pelo Teste de Aprendizagem Auditiva Verbal de Rey (Tabela 3). A comparação dos resultados relativos à Figura Complexa de Rey-Osterrieth, com outros estudos não foi possível, por diferença de metodologia empregada. A maioria das pesquisas utilizando esse teste era tipo caso-controle, não informava o percentual de pacientes com EM e comprometimento de memória de trabalho ou de curto prazo e tinha análise estatística por regressão multivariada ou correlação (Inglese et al., 2008; Schulz et al., 2006).

O percentual de perda de memória de curto prazo avaliada pelo teste RAVLT igual a $17,8 \%$ desta pesquisa foi menor que os 33\% referidos por Schulz et al. (2006), usando o mesmo teste, embora se deva considerar que o padrão não foi construído para a população brasileira. Simioni, Ruffieux, Bruggiman, Annoni e Schluep (2007), ao avaliarem 127 pacientes com diagnóstico de EM, EDSS variando entre zero e 2,5 , sendo $56,7 \%$ na forma surto/ remissão, por meio do RAVLT, identificaram $23,6 \%$ com perda de memória de curto prazo e atribuíram essa perda ao fato de o teste exigir um esforço que pode ser intenso para esses pacientes. Ressaltaram que a perda de memória de curto prazo deve ser valorizada no tratamento da EM, porque compromete muito o desempenho das ativi- 
dades diárias e reduz a qualidade de vida. Inglese et al. (2008), também empregando esse instrumento, informaram que $50 \%$ dos pacientes tinham perda de memória de curto prazo. O percentual de pacientes da presente pesquisa com prejuízo dessa memória, avaliada pelo RAVLT, foi menor que os $33,3 \%$ relatados por Negreiros et al. (2008), testando 54 pacientes na forma clínica surto-remissão, mas teve em comum a identificação de uma curva de aprendizagem que não se associou à gravidade de esclerose múltipla, avaliada pelo EDSS. No estudo de Jønsson et al. (2006), os resultados do teste de dígitos em ordem direta e inversa indicaram que $28,8 \%$ dos pacientes com EM tinham redução de memória operacional, percentual muito menor que os $57,1 \%$ da presente pesquisa.

Tomando em conjunto os resultados da avaliação da função de memória, os prejuízos para memória de trabalho e de curto prazo foram mais freqüentes que os de memória operacional (Tabela 3). No passado, admitiu-se que a redução de memória era causada por distúrbios de evocação da informação. As pesquisas mais recentes sugerem que o comprometimento da memória emerge de prejuízo nos processos de armazenamento da informação e decodificação (Benedict \& Zivadinov, 2006; Diamond, Johnson, Kaufman, \& Graves, 2008; Griffiths et al., 2005). Dessa forma, talvez as cinco tentativas de aprendizagem do RAVLT tenham sido insuficientes para os pacientes com EM codificarem a informação e recordarem (Winkelmann et al., 2007). Do ponto de vista prático, os prejuízos de memória de trabalho e de curto prazo podem comprometer o desempenho de tarefas rotineiras, o que acarreta desconfortos domésticos, porque as pessoas percebem essas falhas como desatenção.

Moriarty et al. (1999), empregando imagem por ressonância magnética, identificaram que lesões justacorticais, ainda que pequenas, têm implicações prognósticas no comprometimento da memória de trabalho, avaliada pelo teste RAVLT, que se agrava com a evolução da doença, mesmo após remissão completa de surtos da forma clínica surto/remissão, devido ao dano neuronal das fibras longas de interconexão cortical que causam síndrome de desconexão. A característica dessa síndrome é um comprometimento da recuperação da memória de curto prazo, mesmo em presença de aprendizagem normal. Essa perda é especialmente importante quando as lesões ocupam a substância branca e a cinzenta do lobo frontal esquerdo, assim como na corona radiata, na cápsula interna, na ínsula e no hipocampo do lobo temporal, dificultando ao cérebro codificar novas associações verbais.

$\mathrm{Na}$ presente pesquisa, $53,5 \%$ dos pacientes tinham fadiga em grau baixo ou alto, percentual próximo ao limite inferior do intervalo de $53 \%$ a $92 \%$ referido por diversos autores (Bol, Duits, Hupperts, Vlaeyen, \& Verhey, 2009). Tanto a fadiga cognitiva quanto a psicossocial associaram-se significantemente ao baixo rendimento de memória no RAVLT dos pacientes analisados (Tabela 3), o que pareceu corroborar a complexa relação entre fadiga e me- mória que Simioni et al. (2007) também relataram. Essa hipótese foi reforçada ao se identificar que apenas as perdas em memória de curto prazo tinham associação significante com fadiga, diferindo dos demais testes empregados para avaliação de memória.

A fadiga física acarreta dificuldades de socialização e desenvolvimento das tarefas do dia-a-dia em casa e no trabalho, o que pode também explicar o achado significante de fadiga psicossocial em portadores de esclerose múltipla com maior tempo de doença e nos mais idosos, assim como uma associação fraca do tempo de doença com fadiga geral e física, a qual deve ser melhor investigada com amostras maiores. Em verdade, a comprovação estatística de fadiga psicossocial veio reforçar uma constatação subjetiva feita durante a aplicação dos testes. Alguns pacientes referiram dificuldades de relacionamento social, configuradas pela evitação em participar de eventos e encontros com amigos, caracterizando um afastamento. Esse fato reforça a necessidade de o profissional de saúde olhar para o doente e considerar seu contexto, e não apenas para a doença, buscando contatar com seu mundo subjetivo, principalmente porque não se pode negar ao paciente a vivência plena como ser biopsicossocial.

Apesar dessa associação, a presença de fadiga física, por si só, não explica a fadiga cognitiva ou mental objetiva, quando avaliada por diversos instrumentos, assim como não parece relacionada com problemas de atenção e concentração. Sentimentos de afetividade negativa, também denominada neuroticismo ou instabilidade emocional, ou seja, uma disposição de experimentar prejuízos psicológicos ao longo do tempo, em diversas situações, pode comprometer o desempenho em testes de cognição (Bol et al., 2009).

Quanto a percepção visual e organização visuoespacial, avaliadas pela cópia da Figura Complexa de ReyOsterrieth, constatou-se que $21,4 \%$ dos pacientes apresentavam perdas. Essas reduções foram mais freqüentes que os $17,9 \%$ referidos por Jønsson et al. (2006). Esses autores alertaram para o fato de que essa redução de percepção melhora quando os mesmos testes são aplicados mais de uma vez aos pacientes, pela redução da ansiedade. No entanto esse procedimento não foi empregado na presente pesquisa.

\section{Conclusões}

A partir da investigação de comprometimento cognitivo em pacientes com esclerose múltipla, concluiu-se que: 21,4\% apresentaram alteração da percepção visual e da organização visuoespacial avaliada por meio da cópia do teste de Rey-Osterrieth; 89,2\% tinham redução da memória de trabalho, avaliada na segunda fase do mesmo teste, e $85,7 \%$ tinham perda de memória de curto prazo detectada na terceira fase. Também foram constatados comprometimento da memória de curto prazo em 17,8\% dos pacientes, na avaliação do RAVLT, e operacional, em 
$57,1 \%$ dos pacientes por meio do teste de dígitos. Quanto à atenção concentrada e sustentada, $57,1 \%$ dos pacientes foram identificados como portadores de perda, pelo teste d2. Por meio do inventário MFIS-21Br, caracterizou-se presença de fadiga de baixo ou alto grau em $53,6 \%$ dos pacientes.

Da análise de associação entre os resultados dos testes neuropsicológicos e as variáveis da esclerose múltipla, constatou-se haver significância entre redução da memória de trabalho, de curto prazo e de atenção concentrada e sustentada em pacientes com idade igual ou maior a 40 anos, assim como entre prejuízo de memória de curto prazo, avaliada pelo RAVLT, e presença de fadiga cognitiva, psicossocial e total, avaliada pelo MFIS-21Br. Concluise também que, dentre as funções cognitivas avaliadas, a memória foi a mais freqüentemente comprometida, seguindo-se a atenção, sendo a percepção comparativamente a mais preservada.

Uma das aplicabilidades dos resultados do presente estudo poderá ser o apoio aos pacientes, por meio da implantação de um programa de reabilitação neurocognitiva no Centro Estadual de Referência, cuja primeira etapa será o estabelecimento da avaliação de atenção, memória e percepção como parte da rotina diagnóstica, seguindose a inclusão dos pacientes com prejuízo em pelo menos uma dessas funções, em atendimento neuropsicológico. As informações derivadas dos testes neuropsicológicos e do próprio acompanhamento reabilitacional poderão ensejar também pesquisas com tamanho amostral maior, possibilitando a comprovação desses resultados.

\section{Referências}

Amato, M. P., Zipoli, V., Goretti, B., Portaccio, E., de Caro, M. F., Ricchiuti, L., et al. (2006). Benign multiple sclerosis. Cognitive, psychological and social aspects in a clinical cohort. Journal of Neurology, 253, 1054-1059.

Andersson, C., Lindau, M., Almkvist, O., Engfeldt, P., Johansson, S. E., \& Jönhagen, M. E. (2006). Identifying patients at high and low risk of cognitive decline using rey auditory verbal learning test among middle-aged memory clinic outpatients. Dementia and Geriatric Cognitive Disorders, 21, 251-259.

Arnett, P., \& Forn, C. (2007). Evaluación neuropsicológica en la esclerosis múltiple. Revista de Neurologia, 44, 166-172.

Balsimelli, S., Mendes, M. F., Bertolucci, P. H. F., \& Tilbery, C. P. (2007). Attention impairment associated with relapsingremitting multiple sclerosis patients with mild incapacity. Arqhives of Neuropsichiatry, 65(2A), 262-267.

Benedict, R. H. B., \& Zivadinov, R. (2006). Predicting neuropsychological abnormalities in multiple sclerosis. Journal of the Neurological Sciences, 245, 62-72.

Benedict, R. H. B., \& Zivadinov, R. (2007). Reliability and validity of neuropsychological screening and assessment strategies in MS. Journal of Neurology, 254(Suppl. 2), II/ $22-\mathrm{II} / 25$

Bol, Y., Duits, A. A., Hupperts, R. M. M., Vlaeyen, J. W. S., \& Verhey, F. R. J. (2009). The psychology of fatigue in patients with multiple sclerosis: A review. Journal of Psychosomatic Research, 66, 3-11.
Boone, K. B., Lu, P., \& Wen, J. (2005). Comparison of various RAVLT scores in the detection of noncredible memory performance. Archives of Clinical Neuropsychology, 20(3), 301-319.

Brickenkamp, R. (2000). Teste d2 - Atenção Concentrada: Manual. São Paulo, SP: Casa do Psicólogo.

Capovilla, A. G. S. (2007). Contribuições da Neuropsicologia Cognitiva e da avaliação neuropsicológica à compreensão do funcionamento cognitivo humano. Cadernos de Psicopedagia, 6(11), 1-24.

Coltheart, M. (2004). Brain imaging, connectionism and Cognitive Neuropsychology. Cognitive Neuropsychology, 21(1), 21-25.

Diamond, B. J., Johnson, S. K., Kaufman, M., \& Graves, L. (2008). Relationships between information processing, depression, fatigue and cognition in multiple sclerosis. Cognitive Neuropsychology, 23, 189-199.

Engel, C., Greim, B., \& Zettl, U. K. (2007). Diagnostics of cognitive dysfunctions in multiple sclerosis. Journal of Neurology, 254(Suppl. 2), II/30-II/34.

Ferreira, M. L. B., Machado, M. I. M., Vilela, M. L., Guedes, M. J., Ataíde, L., Jr., Santos, S., et al. (2004). Epidemiologia de 118 casos de esclerose múltipla com follow-up de 15 anos no Centro de Referência do Hospital da Restauração de Pernambuco. Archives of Neuro-Psichiatry, 62(4), 1027-1032.

Figueiredo, V. L. M., \& Nascimento, E. (2007). Desempenhos nas duas tarefas do subteste dígitos do WISC-III e do WAISIII. Psicologia: Teoria e Pesquisa, 23(3), 313-318.

Goldacre, M. J., Wotton, C. J., Seagroatt, V., \& Yeates, D. (2004). Multiple sclerosis after infectious mononucleosis: Record linkage study. Journal of Epidemioogyl \& Community Health, 58, 1032-1035.

Gonzalez-Rosa, J. J., Vasquez-Marrufo, M., Vaquero, E., Duque, P., Borges, M., Gamero, M. A., et al. (2006). Differential cognitive impairment for diverse forms of multiple sclerosis. BioMed Central Neuroscience, 7, 39.

Griffiths, S. Y., Yamamoto, A., Boudreau, V. G., Ross, L. K., Kozora, E., \& Thornton, A. E. (2005). Memory interference in multiple sclerosis. Journal of International Neuropsychological Society, 11, 737-746.

Inglese, M., Adhya, S., Johnson, G., Babb, J. S., Miles, L., Jaggi, H., et al. (2008). Perfusion lmagnetic resonance imaging correlates of neuropsychological impairment in multiple sclerosis. Journal of Cerebral Blood Flow \& Metabolism, 28(1), 164-171.

Jønsson, A., Andresen, J., Storr, L., Tscherning, T., Sørensen, P. S., \& Ravnborg, M. (2006). Cognitive impairment in newly diagnosed multiple sclerosis patients: A 4-year follow-up study. Journal of Neurological Sciences, 245, 77-85.

Kaplan, E., Fein, D., Morris, R., \& Delis, D. C. (1991). WAIS$R$ as a neuropsychological instrument. San Antonio, TX: The Psychological Corporation.

Kurtzke, J. F., \& Page, W. F. (1997). Epidemiology of multiple sclerosis in US veterans: VII. Risk factors for MS. Neurology, 48, 204-213.

Mäder, M. J. (1996). Avaliação Neuropsicológica: Aspectos históricos e situação atual. Psicologia: Ciência e Profissão, 16(3), 12-18.

McDonald, W. I., Compston, A., Edan, G., Goodkin, D., Hartung, H. P., Lublin, F. D., et al. (2001). Recommended diagnostic criteria for multiple sclerosis: Guidelines from the International Panel on the Diagnosis of Multiple Sclerosis. Annals of Neurology, 50, 121-127. 
Moriarty, D. M., Blacksha,W. A. J., Talbot, P. R., Griffiths, H. L., Snowden, J. S., Hillier, V. F., et al. (1999). Memory dysfunction in Multiple Sclerosis Corresponds to juxtacortical lesion load on fast fluid-attenuated inversion-recovery MR images. American Journal of Neuroradiology, 20, 1956-1962.

Negreiros, M. A., Mattos, P., Paes, R. A., Landeira-Fernandez, J., \& Alvarenga, R. P. (2008). A brief neuropsychological screening test battery for cognitive dysfunction in Brazilian multiple sclerosis patients. Brain Injury, 22(5), 419-426.

Noronha, A. P. P., Sisto, F. F., Bartholomeu, D., Lamounier, R., \& Rueda, F. J. M. (2006). Atenção sustentada e concentrada: Construtos semelhantes? Psicologia: Pesquisa \& Trânsito, 2(1), 29-36.

Pavan, K., Schmidt, K., Marangoni, B., Mendes, M. F., Tilbery, C. P., \& Lianza, S. (2007). Adaptação transcultural e validação da escala modificada de impacto de fadiga. Arquivos de Neuro-Psiquiatria, 65(3A), 669-673.

Penner, I. K., Kappos, L., Rausch, M., Opwis, K., \& Radü, E. W. (2006). Therapy-induced plasticity of cognitive functions in MS patients: Insights from fMRI. The Journal of Physiology, 99, 455-462.

Poser, C. M., Paty, D. W, Scheinberg, L. C., McDonald, W. I., Davis, F. A., Ebers, G. C., et al. (1983). New diagnostic criteria for multiple sclerosis: Guidelines for research protocols. Annals of Neurology, 13, 227-231.

Rey, A. (1999). Teste de cópia e de reprodução de memória de figuras geométricas complexas: Manual. São Paulo, SP: Casa do Psicólogo.

Roessler, R. Y., \& RumrilL, P. D., Jr. (2003). Multiple sclerosis and employment barriers: A systemic perspective on diagnosis and intervention. Journal of Prevention Assessment and Reability, 21(1), 17-23.

Rogers, J. M., \& Panegyres, P. K. (2007). Cognitive impairment in multiple sclerosis: Evidence-based analysis and recommendations. Journal of Clinical Neuroscience, 14, 919-927.

Schulz, D., Kopp, B., Kunkel, A., \& Faiss, J. H. (2006). Cognition in the stage multiple sclerosis. Journal of Neurology, 253, 1002-1010.

Simioni, S., Ruffieux, C., Bruggimann, L., Annoni, J. M., \& Schluep, M. (2007). Cognition, mood and fatigue in patients in the early stage of multiple sclerosis. Swiss Medical Weekly, 137, 496-501.

The Consortium of Multiple Sclerosis Centers Health Services Research Subcommittee. (1997). Multiple Sclerosis Quality of Life Inventory: A user's Manual (pp. 23-24). New York.

Winkelmann, A., Engel, C., Apel, C., \& Zettl, U. K. (2007). Cognitive impairment in Multiple Sclerosis. Journal of Neurology, 254(Suppl. 2), II/35-II/42. 